\title{
New data on lithobiomorph centipedes (Chilopoda: Lithobiomorpa: Anopsobiidae, Henicopidae, Lithobiidae) from Kazakhstan
}

\author{
Новые данные о многоножкках-костянках (Chilopoda: \\ Lithobiomorpha: Anopsobiidae, Henicopidae, Lithobiidae) \\ Респубцики Казахстан
}

\author{
Yu.V. Dyachkov \\ Ю.В. Аьячковов
}

Altai State University, Lenin Avenue, 61, Barnaul 656049, Russia. E-mail: dyachkov793@mail.ru Алтайский государственный университет, проспект Ленина, 61, Барнаул 656049 Россия. stan.

KEY WORDS: Lithobiomorpha, Anopsobiidae, Henicopidae, Lithobiidae, faunistics, new records, Kazakh-

КЛЮЧЕВЫЕ СЛОВА: Lithobiomorpha, Anopsobiidae, Henicopidae, Lithobiidae, фаунистика, новые находки, Казахстан.

ABSTRACT. 11 species of lithobiomorph centipedes are recorded in Kazakhstan: Dzhungaria gigantea Farzalieva, Zalesskaja et Edgecombe, 2004, Cermatobius kirgisicus (Zalesskaja, 1972), Australobius magnus (Trotzina, 1894), Disphaerobius loricatus (Sseliwanoff, 1881), Hessebius golovatchi Farzalieva, 2017, H. multicalcaratus Folkmanová, 1958, H. perelae Zalesskaja, 1978, H. cf. plumatus Zalesskaja, 1978, Lithobius (L.) forficatus (Linnaeus, 1758), L. (Monotarsobius) franciscorum Dányi et Tuf, 2012, and L. (M.) insolens Dányi et Tuf, 2012. Three species are new to the Almaty Region: D. loricatus, $H$. cf. plumatus and L. forficatus. L. forficatus and the genus Lithobius Leach, 1814, as well as the family Lithobiidae and the order Lithobiomorpha, are also new to the fauna of Kyzylorda Region. Remarks are provided and the mouthparts are illustrated for some species encountered. All new records are mapped. A table showing the distribution of all lithobiomorph species in Kazakhstan is provided.

How to cite this article: Dyachkov Yu.V. 2019. New data on lithobiomorph centipedes (Chilopoda: Lithobiomorpa: Anopsobiidae, Henicopidae, Lithobiidae) from Kazakhstan // Arthropoda Selecta. Vol.28. No.1. P.8-20. doi: 10.15298/arthsel. 28.1.02

РЕЗЮМЕ. 11 видов многоножек-костянок зарегистрированы в Казахстане: Dzhungaria gigantea Farzalieva, Zalesskaja et Edgecombe, 2004, Cermatobius kirgisicus (Zalesskaja, 1972), Australobius magnus (Trotzina, 1894), Disphaerobius loricatus (Sseliwanoff, 1881), Hessebius golovatchi Farzalieva, 2017, H. multicalcaratus Folkmanová, 1958, H. perelae Zalesskaja, 1978, H. cf. plumatus Zalesskaja, 1978, Lithobius (L.) forficatus (Linnaeus, 1758), L. (Monotarsobius) franciscorum Dányi et Tuf, 2012 и L. (M.) insolens Dányi et Tuf, 2012. Три вида: D. loricatus, $H$. cf. plumatus и L. forficatus впервые отмечены в Алматинской области. L. forficatus, род Lithobius Leach, 1814, семейство Lithobiidae и отряд Lithobiomorpha впервые отмечены в Кызылординской области. Для некоторых видов приведены примечания и проиллюстрированы ротовые придатки. Дана карта, иллюстрирующая новые находки в регионе. Приводится таблица с указанием распространения всех видов костянок в Казахстане.

\section{Introduction}

Knowledge of the lithobiomorph centipede fauna of Kazakhstan is extensive, but this huge territory is prospected very fragmentarily [Sseliwanoff, 1881; Attems, 1904; Lignau, 1929; Zalesskaja, 1978; Eason, 1997; Farzalieva et al., 2003, 2004, 2017; Farzalieva, 2006, 2017; Tuf, 2007, 2010; Vsevolodova-Perel, 2009; Bragina, 2012, 2016; Dányi, Tuf, 2012; Dyachkov et al., 2016; Dyachkov, 2017a; Dyachkov, Farzalieva, 2018]. To date, 34 species of lithobiomorph centipedes are known from Kazakhstan, mostly from the Almaty and East Kazakhstan regions.

\section{Material and methods}

This paper is based on the material collected in eastern and southern Kazakhstan in 2015-2018 (Map). Specimens were taken by hand and preserved in $70 \%$ ethanol.

Specimens were photographed using an Olympus XC50 camera attached to an Olympus BX51 stereo microscope. Mouthparts, forcipules and legs were mounted in permanent preparations in euparal. 


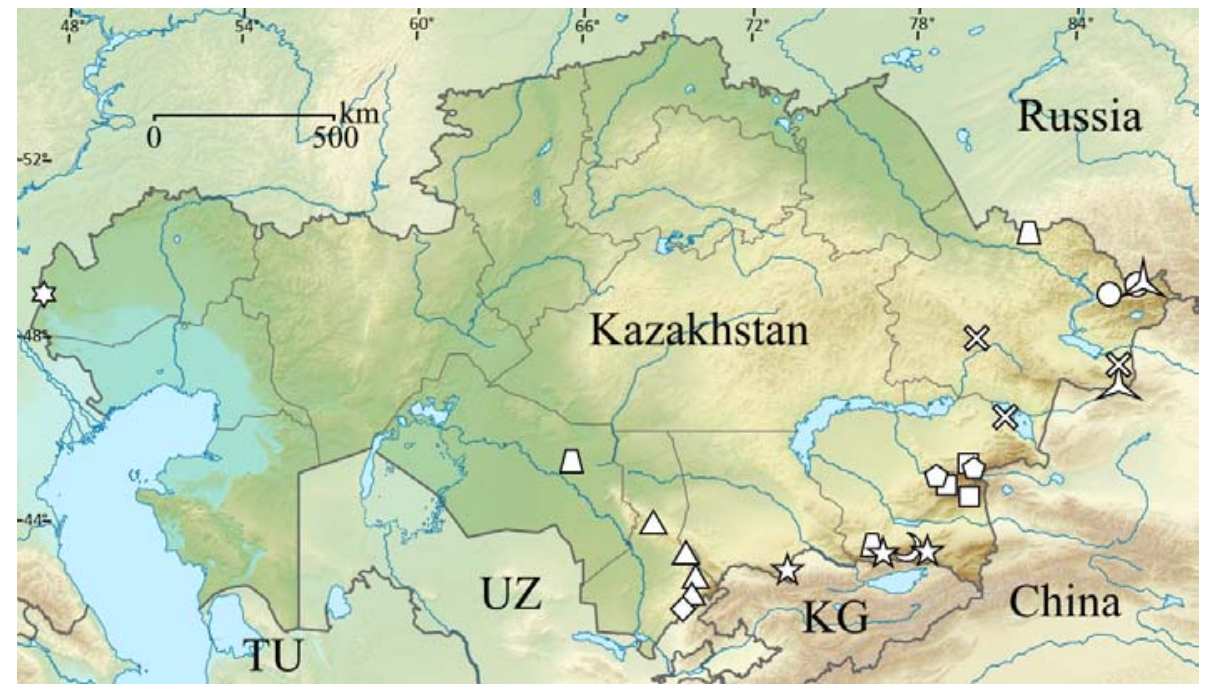

Map. Distribution of Australobius magnus (Trotzina, 1894) (asterisk), Cermatobius kirgisicus (Zalesskaja, 1972) (diamond), Disphaerobius loricatus (Sseliwanoff, 1881) (cross), Dzhungaria gigantea Farzalieva, Zalesskaja, Edgecombe, 2004 (square), Hessebius multicalcaratus Folkmanová, 1958 (six-pointed asterisk), H. perelae Zalesskaja, 1978 (triangle), H. cf. plumatus Zalesskaja, 1978 (crescent), H. golovatchi Farzalieva, 2017 (pentagon), Lithobius (Monotarsobius) insolens Dányi et Tuf, 2012 (three-pointed asterisk), L. (M.) franciscorum Dányi et Tuf, 2012 (circle) and Lithobius forficatus (Linnaeus, 1758) (trapeze) in Kazakhstan.

Карта. Распространение Australobius magnus (Trotzina, 1894) (пятиконечная звезда), Cermatobius kirgisicus (Zalesskaja, 1972) (ромб), Disphaerobius loricatus (Sseliwanoff, 1881) (крестик), Dzhungaria gigantea Farzalieva, Zalesskaja, Edgecombe, 2004 (четырехугольник), Hessebius multicalcaratus Folkmanová, 1958 (шестиконечная звезда), H. perelae Zalesskaja, 1978 (треугольник), $H$. сf. plumatus Zalesskaja, 1978 (полумесяц), H. golovatchi Farzalieva, 2017 (пятиугольник), Lithobius (Monotarsobius) insolens Dányi et Tuf, 2012 (трехконечная звездочка), L. (M.) franciscorum Dányi et Tuf, 2012 (круг) и Lithobius forficatus (Linnaeus, 1758) (трапеция) в Казахстане.

The material is shared between the collections of the Altai State University, Barnaul (ASU), the Zoological Museum of the Moscow State University, Moscow (ZMMU), the Perm State University, Perm (PSU).

The terminology for external anatomy follows that of Bonato et al. [2010]. Body length was measured from the anterior margin of the cephalic plate to the posterior end of the postpedal tergite. The following abbreviations are used in the text, table and maps: morphology: T, TT - tergite, tergites, $\mathrm{D}$ - dorsal, $\mathrm{Tr}$ - trochanter, $\mathrm{P}$ - prefemur, $\mathrm{F}$ femur, Ti - tibia, Ts - tarsus; names of collectors: RD R.Yu. Dudko, YD - Yu.V. Dyachkov, AF - A.A. Fomichev, GK - G.N. Kuftina, AEN - A.E. Nakonechniy, AN - A.E. Naydenov, EN - E.A. Nepaeva, VR - V.V. Rudoi, IT - I.I. Temreshev, RY - R.V. Yakovlev; countries: TU - Turkmenistan, UZ - Uzbekistan, KG - Kyrgyzstan; regions: AKM - Akmola, AKT - Aktobe, AT Atyrau, AL - Almaty, EK - East Kazakhstan, KA Karaganda, KO - Kostanay, KY - Kyzylorda, MA Mangystau, NK - North Kazakhstan, PR - Pavlodar, SK South Kazakhstan, WK - West Kazakhstan, JA — Jambyl Regions.

\section{New records}

Family ANOPSOBIIDAE Verhoeff, 1907

Genus Dzhungaria Farzalieva, Zalesskaja et Edgecombe, 2004
Dzhungaria gigantea Farzalieva, Zalesskaja et Edgecombe, 2004 Map.

Dzhungaria gigantea Farzalieva, Zalesskaja et Edgecombe, 2004: 223; Koch, Edgecombe, 2008: 4-19; Zapparoli, Edgecombe, 2011: 377; Shear, 2018: 280.

MATERIAL. Almaty Region, Dzungarian Alatau Mts: $1 \sigma^{7}$ (ASU No. 60), between Aksu and Krasnyi Yar rivers, stony steppe with shrub thickets, N4506'00.13", E79³4'59.93", 1700-1800 m a.s.1., 22.VI.2016; $1 \sigma^{7}, 1$ ㅇ (ASU No. 61), Burkhansartay Mt. Range, N44³4'00.18", E79 ${ }^{\circ} 7^{\prime} 00.09^{\prime \prime}, 3000 \mathrm{~m}$ a.s.1., 27.VI.2016; 1 ( ASU No. 62), Ermenesay River Valley, stony meadow, N44응

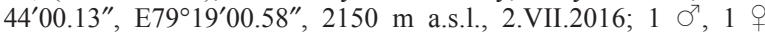
(ASU No. 63), same place, stony mountain tundra, N44 $43^{\prime} 00.10^{\prime \prime}$, E79 ${ }^{\circ} 18^{\prime} 00.12^{\prime \prime}, 3000-3100 \mathrm{~m}$ a.s.1., 3.VII.2016; $1 \sigma^{7}$ (ASU No. 64), between Ermenesay and Koksay rivers, stony alpine meadow,

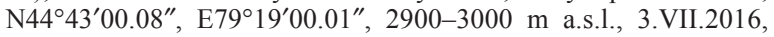
all AF.

DISTRIBUTION. This species is known only from the Dzungarian Alatau [Farzalieva et al., 2004] and has never been recorded above $1800 \mathrm{~m}$ a.s.l.

REMARKS. Body length 12-22 mm (vs. 13-15 mm in the original description); 33-43 antennomeres (vs. 30-38 in the original description).

COMMENTS. The existing opinions concerning the taxonomic position of this genus and species are varied [Farzalieva et al., 2004; Koch, Edgecombe, 2008; Zapparoli, Edgecombe, 2011; Bonato et al., 2016; Shear, 2018]. Farzalieva et al. [2004] and Zapparoli \& Edgecombe [2011: 377] identified this genus as a member of Anopsobiidae, vs. Koch \& 


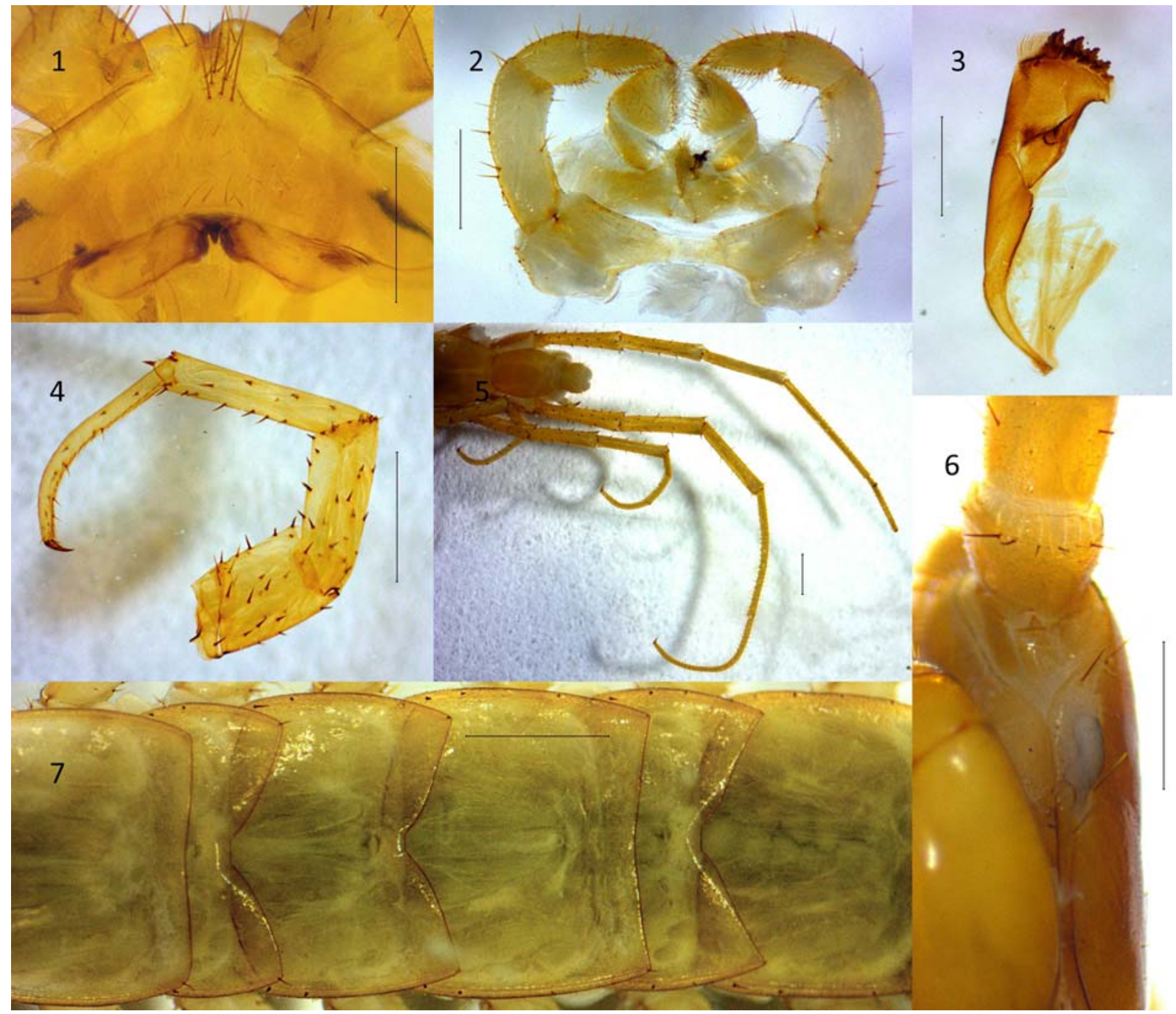

Figs 1-7. Cermatobius kirgisicus (Zalesskaja, 1972): 1 - clypeus and labrum, ventral view; 2 - maxillary complex, ventral view; 3 mandible; 4 - leg 5, lateral view; 5 - ultimate legs of female, dorsal view; 6 - ocellus, lateral view; 7 - tergites 5-10, dorsal view. Scale: $1-3-0.25 \mathrm{~mm}, 4,5,7-1 \mathrm{~mm}, 6-0.5 \mathrm{~mm}$.

Рис. 1-7. Cermatobius kirgisicus (Zalesskaja, 1972): 1 - клипеус и лабрум, вентрально; 2 - максиллярный комплекс, вентрально; 3 - мандибула; 4 - 5-я нога, латерально; 5 - последняя пара ног самки, дорсально; 6 - глазок, латерально; 7 - 510 тергиты, дорсально. Масштаб: $1-3-0,25$ мм, 4, 5, $7-1$ мм, $6-0,5$ мм.

Edgecombe [2008] and Bonato et al. [2016] who consider this genus as a henicopid.

\section{Family HENICOPIDAE Pocock, 1901}

\section{Genus Cermatobius Haase, 1885}

\section{Cermatobius kirgisicus (Zalesskaja, 1972)} Map, Figs 1-7.

Cermatobius kirgisicus (Zalesskaja, 1972): 608-611; 1978: 25-27; Eason, 1997: 120-121.

MATERIAL. South Kazakhstan Region, western Tian-Shan: $3 \bigcirc^{\top} \bigcirc^{\top}, 5$ Oᄋ, 4 juv. (ASU No. 65), Ugamskiy Mt. Range, SayramUgam National Park, Sazanata River Valley, Betula, N42 $09^{\prime} 00.10^{\prime \prime}$,

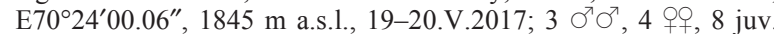
(ASU No. 66), same valley, N42 $10^{\prime} 00.21^{\prime \prime}$, E70 $25^{\prime} 00.07^{\prime \prime}$, ca $1955 \mathrm{~m}$ a.s.1., 3-6.VI.2017, all YD.
DISTRIBUTION. C. kirgisicus is known from Kazakhstan, Kyrgyzstan and Uzbekistan [Zalesskaja, 1978; Eason, 1997]. Kazakhstan: South Kazakhstan Region (western TianShan: Aksu-Zhabagly Nature Reserve [Zalesskaja, 1978] and Sayram-Ugam National Park).

REMARKS. According to Zalesskaja [1972, 1978] and Eason [1997], this species has 41-71 antennal articles (5375 in the studied specimens); $5+5-7+7$ short coxosternal teeth, often unequal on either side, e.g. $7+8(5+5-8+8$ in the studied specimens); from $3+4$ to $5+5-6+6$ spurs of the female gonopods $(4+4-5+5$ in the above specimens). Ocellus, maxillary complex, mandible, clypeus and labrum, 5-10 TT, $5 \mathrm{leg}$ and ultimate legs are as in Figs 1-7.

\section{Family LITHOBIIDAE Newport, 1844}

Genus Australobius Chamberlin, 1920 


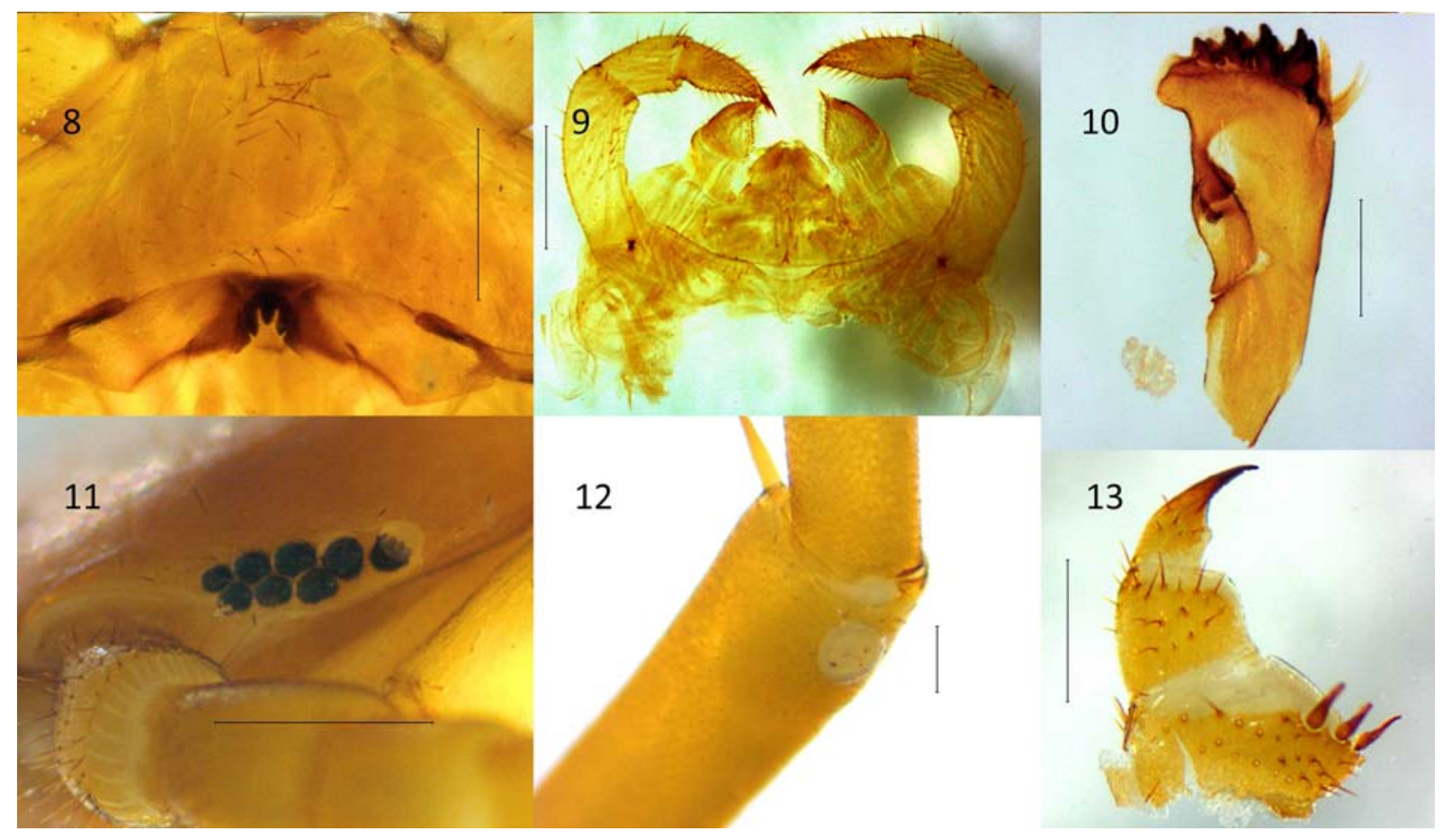

Figs 8-13. Australobius magnus (Trotzina, 1894): 8 - clypeus and labrum, ventral view; 9 - maxillary complex, ventral view; 10 mandible; 11 - ocelli, lateral view; 12 - wart-shape process on male tibia 15, dorsal view; 13 - female gonopod, lateral view. Scale: 8 , 9, $11-0.5 \mathrm{~mm}, 10,13-0.25 \mathrm{~mm}, 12-0.12 \mathrm{~mm}$.

Рис. 8-13. Australobius magnus (Trotzina, 1894): 8 - клипеус и лабрум, вентрально; 9 - максиллярный комплекс, вентрально; 10 - мандибула; 11 - глазки, латерально; 12 - бородавкоподобный вырост на 15 -й голени самца, дорсально; 13 - гонопод самки, латерально. Масштаб: 8, 9, $11-0,5$ мм, 10, $13-0,25$ мм, $12-0,12$ мм.

\section{Australobius magnus (Trotzina, 1894) Map, Figs 8-13.}

Australobius magnus (Trotzina, 1894): 248; Zalesskaja, 1978: 142; Eason, 1989: 368; Eason, 1997: 120.

MATERIAL. 1 ○ (ASU No. 58), Jambyl Region, Kyrgyz Ala-Too Mt. Range, Merke River Valley, near Esenkulsay River estuary, forest, N42 $43^{\prime} 56^{\prime \prime}$, E73 $13^{\prime} 33^{\prime \prime}, 1180 \mathrm{~m}$ a.s.1., 15.VIII.2017, AF, YD; $2 \sigma^{7} \sigma^{7}, 2$ juv. (ASU No. 59), Almaty Region, Küngöy Ala-Too Mt. Range, near an unnamed tributary of Kolsay River, between Kolsay-1 and Kolsay-2 lakes, Pícea forest, under stones, in moss, under dead wood, N42 ${ }^{\circ} 7^{\prime} 36^{\prime \prime}$, E78 $18^{\prime} 45^{\prime \prime}, 1870 \mathrm{~m}$ a.s.1., 21-22.VIII.2017, YD; $1 \sigma^{\top}$ (PSU), same Region, Uygur District, Kyrgyzsay village, mountain steppe, 12.VII.1978.

DISTRIBUTION. This species seems to be widespread in Central Asia: Kazakhstan, northern Tian-Shan (Almaty (Trans-Ili Alatau and Küngöy Ala-Too Mt. ranges) and Jambyl (Kyrgyz Ala-Too Mt. Range) regions), Tajikistan, Kyrgyzstan [Trotzina, 1894; Attems, 1904; Lignau, 1929; Zalesskaja, 1978; Eason, 1997; Dyachkov, 2017a]. In addition, it is known from China [Ma et al., 2014] and eastern Nepal [Eason, 1989, 1997]. The record from Georgia, Caucasus (Batumi) [Attems, 1907] is doubtful [Zalesskaja, 1978: 144].

REMARKS. Ocelli, clypeus, labrum, maxillary complex, mandible, a wart-shaped process on male $15 \mathrm{Ti}$ and female gonopod as in Figs 8-13.

Genus Disphaerobius Attems, 1926

Disphaerobius loricatus (Sseliwanoff, 1881) Map, Figs 14-19.
Disphaerobius loricatus (Sseliwanoff, 1881): 16; Zalesskaja, 1978: 119; Farzalieva, Zalesskaja, 2003: 265-266; Farzalieva et al., 2017: 131-134.

MATERIAL. 1 (ASU No. 83), East Kazakhstan Region, Saur Mts, $20 \mathrm{~km}$ SEE Zaisan village, N47 $22^{\prime} 00.20^{\prime \prime}$, E85 $09^{\prime}$ 00.22", 1225-1250 m a.s.1., 20.VI.2018, RY, VR, AN; $10^{7}$ (PSU), same Region, Kalkutty River Valley, 32 km NW Ayagoz City, salina near river, $\mathrm{N} 48^{\circ} 10^{\prime}$, E $80^{\circ} 04^{\prime}, 770 \mathrm{~m}$ a.s.1., 15.IV.2016, AF, AN, RD; 1 juv. (PSU), Almaty Region, near Saykan station, sandy steppe, N462 $26^{\prime}, \mathrm{E} 80^{\circ} 41^{\prime}, 400 \mathrm{~m}$ a.s.l., 15.IV.2016, AF, AEN, RD.

DISTRIBUTION. Known from Russia, Orenburg Region (Sol-Iletsk District) in the west to the East Kazakhstan Region in the east [Sseliwanoff, 1881; Zalesskaja, 1978; Farzalieva, Zalesskaja, 2003; Farzalieva et al., 2017]. In Kazakhstan: Aktobe, East Kazakhstan [Farzalieva et al., 2017] and Almaty regions.

REMARKS. Based on the main characters, all above specimens belong to this species, but the sole female studied has the shoulders of the forcipular coxosternite sloping more strongly than illustrated by Farzalieva et al. [2017]. This species is new to the centipede list of the Almaty Region. Clypeus, labrum, maxillary complex, mandible, forcipules and female gonopods as in Figs 14-19.

\section{Genus Hessebius Verhoeff, 1941}

Hessebius golovatchi Farzalieva, 2017 Map, Figs 20-23.

Hessebius golovatchi Farzalieva, 2017: 30-32.

MATERIAL. Almaty Region, Dzungarian Alatau Mts: $1 \sigma^{7}$ (ASU No. 45), Aksu River (near source of Akambay River), stony 


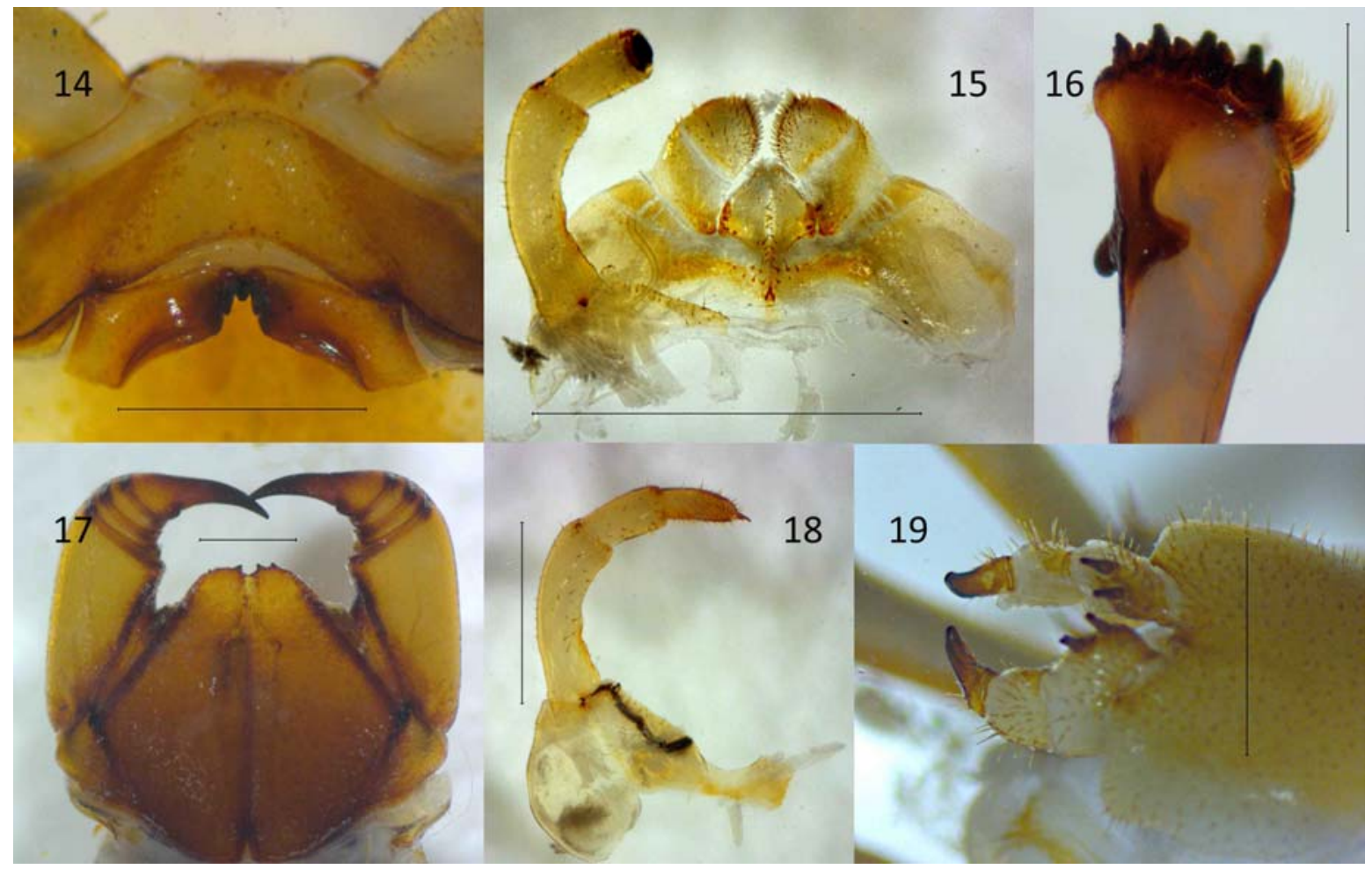

Figs 14-19. Disphaerobius loricatus (Sseliwanoff, 1881): 14 - clypeus and labrum, ventral view; 15 - first maxillae, ventral view; 16 - mandible; 17 - forcipules, ventral view; 18 - left part of second maxillae, dorsal view; 19 - female gonopods, ventrolateral view. Scale: $14,15,17-19-1 \mathrm{~mm}, 16-0.5 \mathrm{~mm}$

Рис. 14-19. Disphaerobius loricatus (Sseliwanoff, 1881): 14 - клипеус и лабрум, вентрально; 15 - первые максиллы, вентрально; 16 - мандибула; 17 - ногочелюсти, вентрально; 18 - левая часть вторых максилл, дорсально; 19 - гоноподы самки, вентролатерально. Масштаб: 14, 15, 17-19-1 мм, 16 - 0,5 мм.

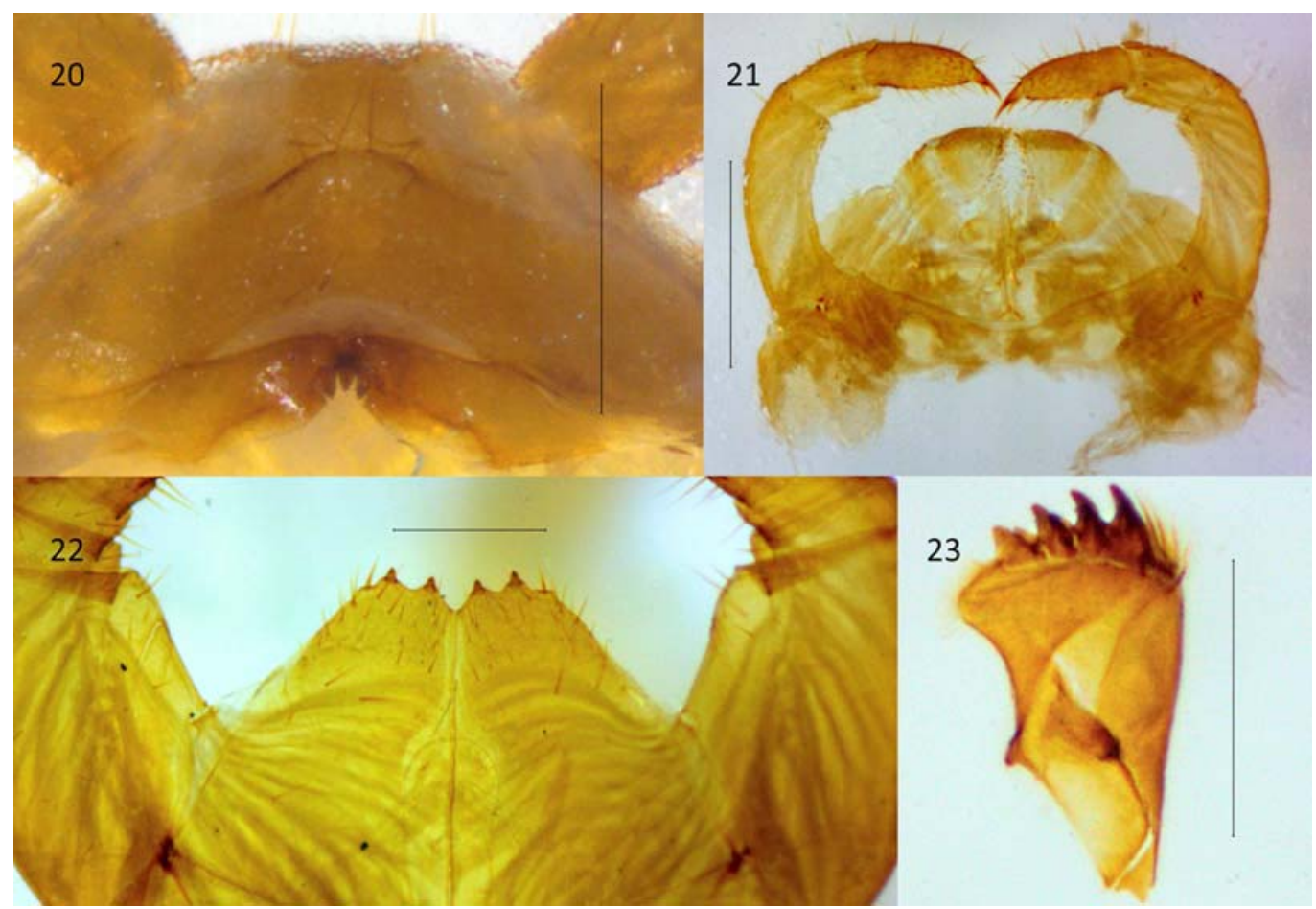

Figs 20-23. Hessebius golovatchi Farzalieva, 2017: 20 - clypeus and labrum, ventral view; 21 - maxillary complex, ventral view; 22 - dental margin of forcipular coxosternite, ventral view; $23-$ mandible. Scale: $20,21-0.5 \mathrm{~mm}, 22,23-0.25 \mathrm{~mm}$.

Рис. 20-23. Hessebius golovatchi Farzalieva, 2017: 20 - клипеус и лабрум, вентрально; 21 - максиллярный комплекс, вентрально; 22 - зубной край коксостернита ногочелюсти, вентрально; 23 - мандибула. Масштаб: $20,21-0,5$ мм, 22, 23 - 0,25 мм. 


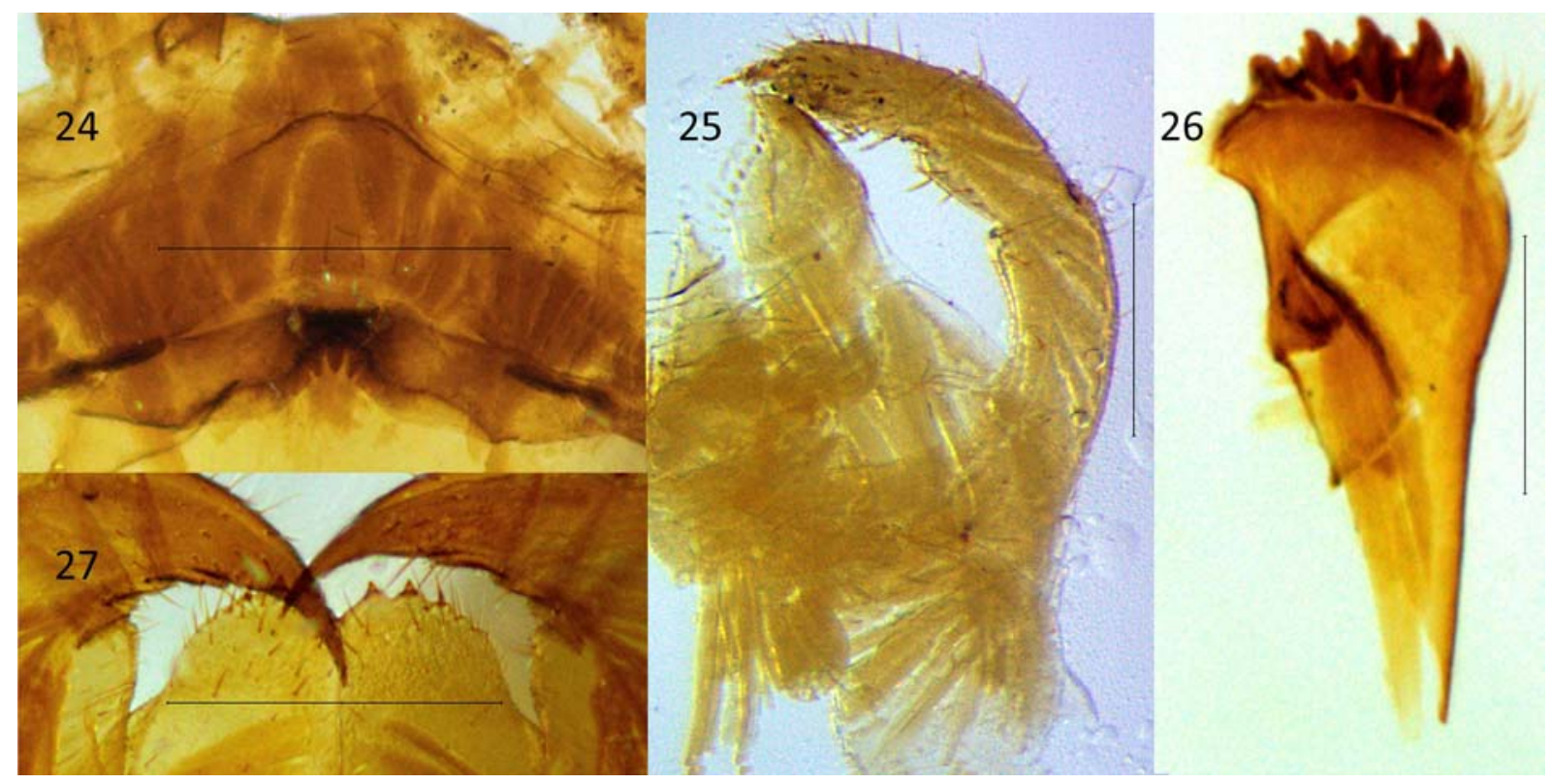

Figs 24-27. Hessebius multicalcaratus Folkmanová, 1958: 24 - clypeus and labrum, ventral view; 25 - left part of maxillary complex, ventral view; 26 - mandible; 27 - dental margin of forcipular coxosternite, ventral view. Scale: $24,27-0.5 \mathrm{~mm}, 25,26-$ $0.25 \mathrm{~mm}$.

Рис. 24-27. Hessebius multicalcaratus Folkmanová, 1958: 24 - клипеус и лабрум, вентрально; 25 - левая часть максиллярного комплекса, вентрально; 26 - мандибула; 27 - зубной край коксостернита ногочелюсти, вентрально. Масштаб: 24,27 - 0,5 мм, $25,26-0,25 \mathrm{Mm}$

stream, $\mathrm{N}^{\circ} 5^{\circ} 03^{\prime} 00.91^{\prime \prime}, \mathrm{E} 79^{\circ} 40^{\prime} 00.67^{\prime \prime}, 3000-3300 \mathrm{~m}$ a.s.1., 23.VI. 2016; 1 (ASU No. 44), between Ermenesay and Koksay rivers,

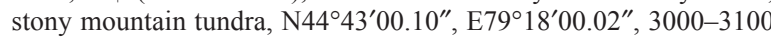
m a.s.1., 3.VII.2016; 2 O $^{7}, 3$ 3 (ASU No. 43), same place, stony

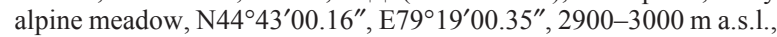
3.VII.2016, all AF. 2017].

DISTRIBUTION. Dzungarian Alatau Mts [Farzalieva,

REMARKS. Most of the studied specimens have 20 antennal articles. Clypeus, labrum, maxillary complex, mandible, dental margin of forcilupar coxosternite as in Figs 20-23.

\section{Hessebius multicalcaratus Folkmanová, 1958 Map, Figs 24-27.}

Hessebius multicalcaratus Folkmanová, 1958: 186; Zalesskaja, 1978: 45-47; Vsevolodova-Perel, 2009: 138; Bragina, 2012: 141; 2016: 109; Kunah, 2013: 73; Zuev, Evsyukov, 2016: 418.

MATERIAL. West Kazakhstan Region, Zhanybek Research Station: 2 우 (ZMMU No. 7798), forest belt, soil sample No. 3, 13.V.1965; 1 ठ, 1 ( (ZMMU No. 7877), same place, soil sample Nos 1 and 6, 12-14.V.1965; $2 \sigma^{\top} \sigma^{7}, 1$ (ZMMU No. 7879), same place, soil sample No. 31, 15.V.1966; 1 ๙, 2 우 (ZMMU No. 7880), same place, slope, soil sample Nos 4-5, 13.V.1965; 1 ठ (ZMMU No. 7882), same place, depression, 15.V.1965; 1 ㄱ, 1 웅 1 fragm. (ZMMU No. 7878), same place, flat salina (aridisol), 6.V.1965; 1 क (ZMMU No. 7883), same place, Matricaria, 1.V.1965, coll. unknown.

DISTRIBUTION. Russia (Volgograd and Rostov-onDon regions), Ukraine (Dnepropetrovsk Region) [Zalesskaja, 1978, Zuev, Evsyukov, 2016]. Kazakhstan (West Kazakhstan and Kostanay regions) [Vsevolodova-Perel, 2009; Bragina, 2012, 2016].

REMARKS. According to Zalesskaja [1978], body length is $9-11 \mathrm{~mm}$ (vs. 9-21 $\mathrm{mm}$ in the above specimens); coxal pores 2-3 (vs. 2-5 in the studied specimens). P, F of 14 and 15 legs of male slightly dilated, 14P and 15P have small D sulci in the studied specimens (not mentioned by Folkmanová [1958] and Zalesskaja [1978] because of the lack of male material). Clypeus, labrum, maxillary complex, mandible and dental margin of forcipular coxosternite as in Figs 24-27.

\section{Hessebius perelae Zalesskaja, 1978 Map, Figs 28-32.}

Hessebius perelae Zalesskaja, 1978: 43-45.

MATERIAL. South Kazakhstan Region: $11 \sigma^{7} \sigma^{7}, 12$ ㅇ (ASU No. 49), $10 \mathrm{~km}$ SW of Abay, Karatau Mt. Range, Karatau Nature Reserve, Kelinschektau Mts, steppe, under stones, N4347'00.13", E68 46'00.02", 1029 m a.s.1., 6-7.V.2017; 2 ๙ $^{\top} \sigma^{\prime}, 4$ 우 (ASU No. 47), same Region, $12 \mathrm{~km}$ E Terekty village, Karatau Mt. Range, Syrdarya-Turkestan State Regional Natural Park, Boralday River Valley, meadow, under stones, N42 $51^{\prime} 00.56^{\prime \prime}$, E69 $51^{\prime} 00.29^{\prime \prime}$, ca

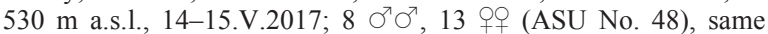
Region, western Tian-Shan Mts, Ugam Mt. Range, Sayram-Ugam National Park, $10 \mathrm{~km}$ NE of Tulkubas village, Iirsu River Valley, meadow, under stones, N42 $24^{\prime} 00.03^{\prime \prime}$, E70 $21^{\prime} 00.11^{\prime \prime}, 1296 \mathrm{~m}$ a.s.1., 16-18.V.2017; $4 \sigma^{7} \sigma^{7}, 1$ ( 1 (ASU No. 46), same Mt. Range, Sayram-Ugam National Park, Sazanata River Valley, steppe, N42 ${ }^{\circ}$ $10^{\prime} 00.10^{\prime \prime}, \mathrm{E} 70^{\circ} 25^{\prime} 00.20^{\prime \prime}, 2035 \mathrm{~m}$ a.s.1., 3-6.VI.2017, all YD.

DISTRIBUTION. South Kazakhstan Region: western Tian-Shan (Aksu-Zhabagly Nature Reserve) and Karatau Mts (Sayram-Ugam National Park [Zalesskaja, 1978], Karatau Nature Reserve, Syrdarya-Turkestan State Regional Natural Park).

REMARKS. The studied male specimens have 1-segmented gonopods with 6-10 setae (vs. $10-12$ in the original description). Clypeus, labrum, maxillary complex, mandible, dental margin of forcipular coxosternite and Tr, P, F of ultimate legs of male as in Figs 28-32. 


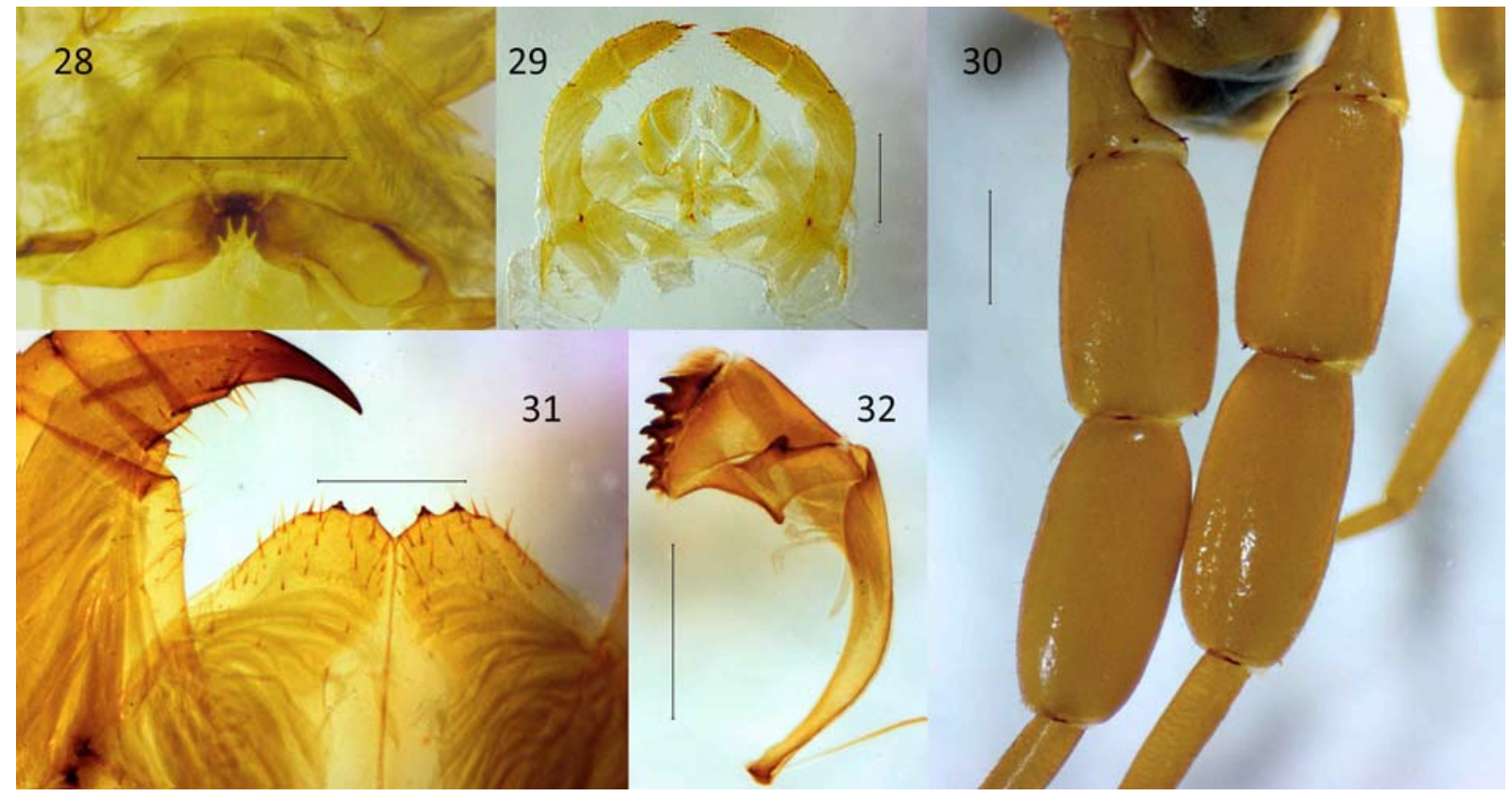

Figs 28-32. Hessebius perelae Zalesskaja, 1978: 28 - clypeus and labrum, ventral view; 29 — maxillary complex, ventral view; 30 trochanter, prefemur and femur of male legs 15, dorsal view; 31 - dental margin of forcipular coxosternite, ventral view; 32 - mandible. Scale $0.5 \mathrm{~mm}$

Рис. 28-32. Hessebius perelae Zalesskaja, 1978: 28 - клипеус и лабрум, вентрально; 29 - максиллярный комплекс, вентрально; 30 - вертлуг, предбедро и бедро 15 ног самца, дорсально; 31 - зубной край коксостернита ногочелюсти, вентрально; 32 мандибула. Масштаб 0,5 мм.

\section{Hessebius cf. plumatus Zalesskaja, 1978 Map, Figs 33-42.}

Hessebius plumatus Zalesskaja, 1978: 47; Bragina, 2012: 141; 2016: 109 .

MATERIAL. Almaty Region: 1 (ASU No. 90), Küngöy AlaToo Mt. Range, between Kolsay-1 and Kolsay-2 lakes, near source of an unnamed tributary of Kolsay River, mountain tundra with stones, N42 ${ }^{\circ} 56^{\prime} 10^{\prime \prime}$, E78 $16^{\prime} 34^{\prime \prime}, 3110-3160 \mathrm{~m}$ a.s.1., 22.VIII.2017, $\mathrm{AF}$.

DISTRIBUTION. H. plumatus is widespread in Central Asia: Kyrgyzstan (Jalal-Abad Region), Tajikistan (Districts of Republican Subordination and Khatlon Region) [Zalesskaja, 1978], Kazakhstan (Kostanay Region) [Bragina, 2012, $2016]$.

REMARKS. The above specimen is extremely close to H. plumatus Zalesskaja, 1978 by all of its main characters, but differs by $3+3$ gonopodal spurs. This difference may reflect a deviation or variability. This species is new to the lithobiomorph centipede list of the Almaty Region. Habitus, dental margin of forcipular coxosternite, ocelli, coxal pores, gonopods and claw of 15Ts as in Figs 33-42.

\section{Genus Lithobius Leach, 1814}

\section{Lithobius (Lithobius) forficatus (Linnaeus, 1758) Map, Figs 43-47.}

Lithobius (Lithobius) forficatus (Linnaeus, 1758): Zalesskaja 1978: 69-71; Farzalieva, 2008: 41-42; Farzalieva et Esyunin, 2008: 926-929; Nefediev et al., 2016: 261-263; Zuev, 2016: 25; Zuev et Evsyukov, 2016: 418-419; Prado et al., 2018: 560-563.

MATERIAL. $3 \sigma^{7} \sigma^{7}$ (ASU No. 50), East Kazakhstan Region,
Shemonaikha town, garden, N50³7'20.16", E81 ${ }^{\circ} 55^{\prime} 04.36^{\prime \prime}, 308$ $\mathrm{m}$ a.s.1., 6.V.2017, VR; 2 +o (ASU No. 51), Almaty Region, Trans-Ili Alatau Mt. Range, Malaya Almatinka River Valley, Medeu, 21.VIII.2015, IT; $1 \sigma^{7}$ (ASU No. 52), same Mt. Range, Kazakchka River Valley, 15.V.2015, IT; 1 ऽ (ASU No. 53), same Mt. Range, Butakovka River Valley, 7.X.2016, IT; 19 (ASU No. 159), same Region, Almaty city, Rakhat District, 7.XI.2018, IT; 1 9 (ASU No. 160), Kyzylorda Region, vicinity of Abay village, paddy fields with Orýza, 26.III.2018, IT.

DISTRIBUTION. This species is widespread through human agency (North and South America, Greenland, Europe, western Siberia and Mongolia) [Zalesskaja, 1978; Farzalieva, 2008; Tuf et al., 2015; Nefediev et al., 2016; Zuev, Evsyukov, 2016; Prado et al., 2018]. In Kazakhstan: East Kazakhstan Region (Zyryanovsk City and Ulken-Naryn village) [Dyachkov et al., 2016], Almaty and Kyzylorda regions.

REMARKS. Clypeus, labrum, maxillary complex, mandible, dental margin of forcipular coxosternite and $11 \mathrm{~T}$ as in Figs 43-47. This species is new to the lithobiid centipede list of the Almaty and Kyzylorda regions.

\section{Lithobius (Monotarsobius) franciscorum Dányi et Tuf, 2012} Map, Figs 48-51.

Lithobius (Monotarsobius) franciscorum Dányi et Tuf, 2012: 16-28; Dyachkov, 2017b: 454; Nefediev et al., 2017a: 220-221.

MATERIAL. East Kazakhstan Region, Katonkaragay District: 1 (ASU No. 18), near Korobikha village, Picea forest, in dead wood, N49 $27^{\prime} 32.4^{\prime \prime}$, E085 $03^{\prime} 14.5^{\prime \prime}$, ca. $600 \mathrm{~m}$ a.s.1., 4.VII. 2017, GK, EN; 1 q (ASU No. 84), Listvyaga Mt. Range, near Aksharbak village, $\mathrm{N}^{\circ} 9^{\circ} 32^{\prime} 01.40^{\prime \prime}$, E085 $32^{\prime} 01.51^{\prime \prime}, 1400 \mathrm{~m}$ a.s.1., 3-5.VII.2018, RY, VR, AN. 

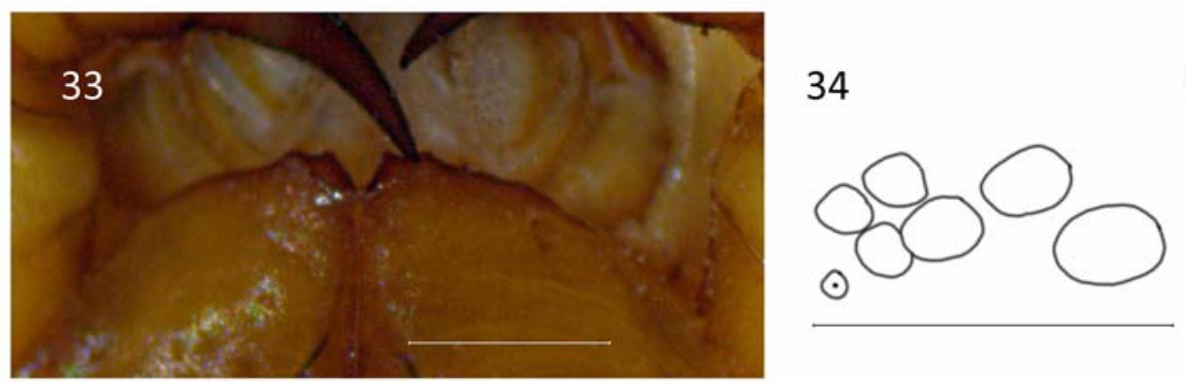

35
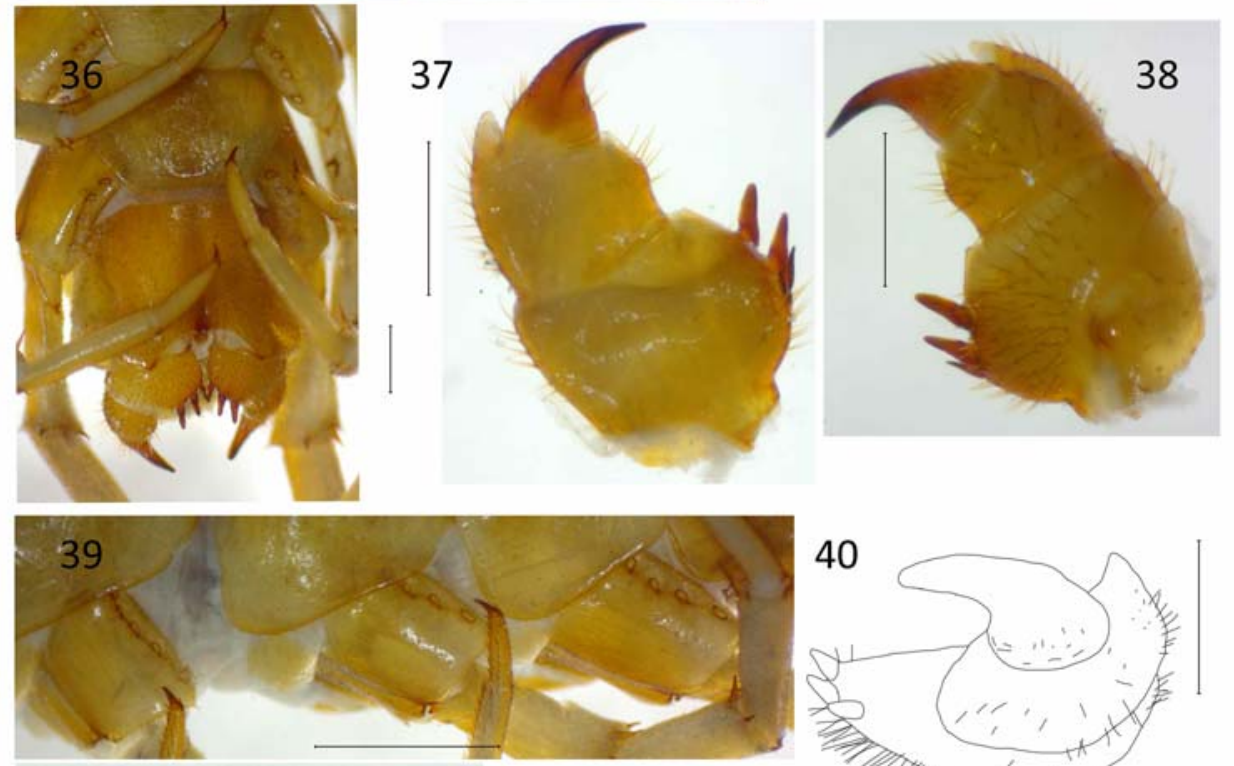

40
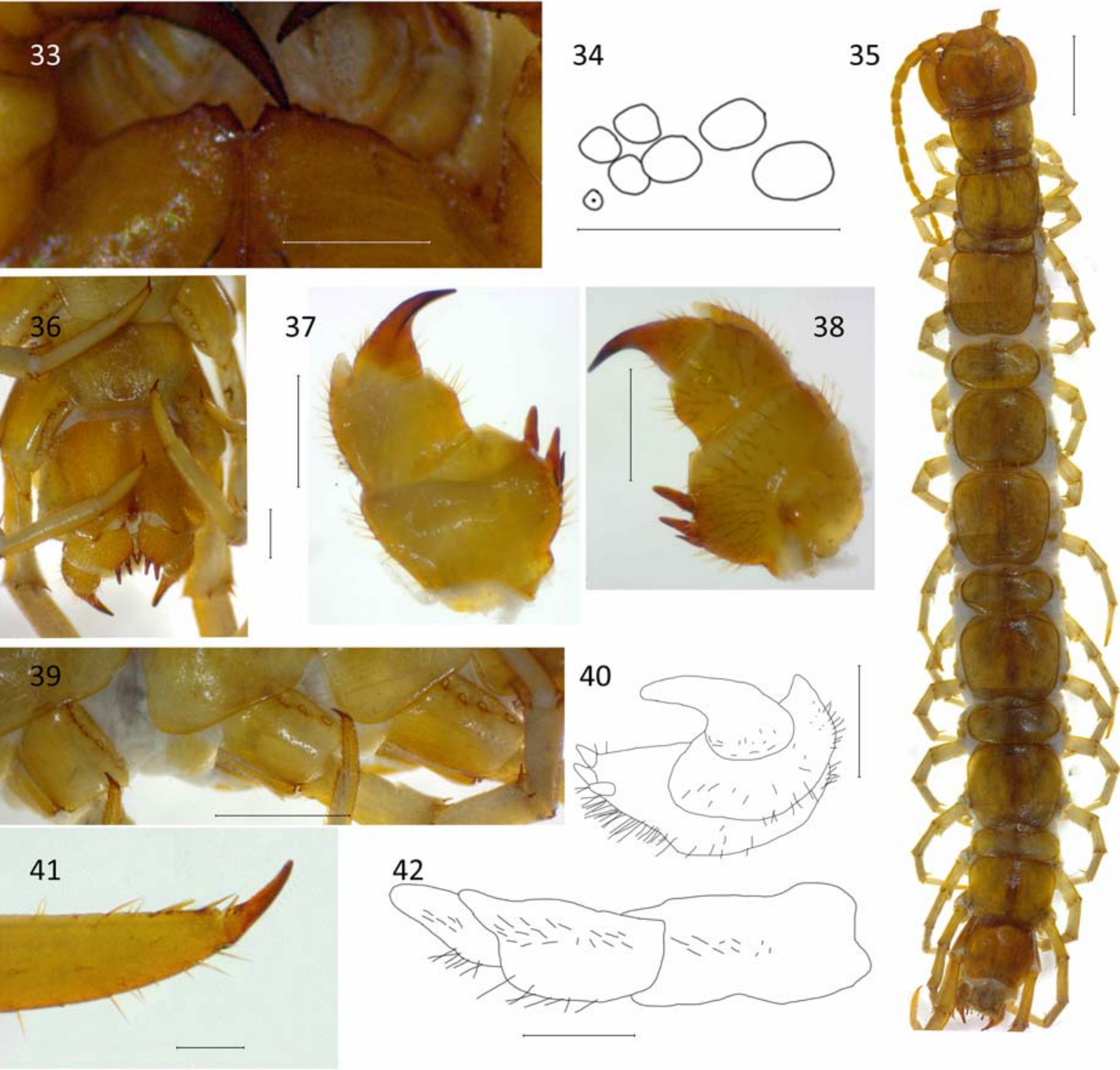

Figs 33-42. Hessebius cf. plumatus Zalesskaja, 1978: 33 - dental margin of forcipular coxosternite, ventral view; 34 - ocelli, lateral view; 35 - habitus, dorsal view; 36 - terminal part of body, ventral view; 37 - female gonopod, mesal view; 38 - same, lateral view; 39 - 12-14 coxae, ventral view; 40 - female gonopod, dorsal view; 41 - claw of tarsus of leg 15; 42 - female gonopod, dorsal view. Scale: $33,34,36-38,40,42-0.5 \mathrm{~mm}, 35-2.5 \mathrm{~mm}, 39-1 \mathrm{~mm}, 41-0.12 \mathrm{~mm}$.

Рис. 33-42. Hessebius cf. plumatus Zalesskaja, 1978: 33 - зубной край коксостернита ногочелюсти, вентрально; 34 — глазки, латерально; 35 - габитус, дорсально; 36 - конечная часть тела, вентрально; 37 - гонопод самки, вид изнутри; 38 - то же, латерально; 39 - 12-14 тазики, вентрально; 40 - гонопод самки, дорсально; 41 - коготь лапки 15 ноги; 42 - гонопод самки, дорсально Масштаб: $33,34,36-38,40,42-0,5$ мм, $35-2,5$ мм, $39-1$ мм, $41-0,12$ мм.

DISTRIBUTION. This species is distributed in the East Kazakhstan Region (Kazakhstan's Altais) [Dányi, Tuf, 2012] and the Republic of Altai [Dyachkov, 2017b; Nefediev et al., 2017a].

REMARKS. Clypeus, labrum, maxillary complex, mandible and dental margin of forcipular coxosternite as in Figs $48-51$.

Lithobius (Monotarsobius) insolens Dányi et Tuf, 2012

Map, Figs 52-56.
Lithobius (Monotarsobius) insolens Dányi et Tuf, 2012: Farzalieva, 2006: 105-108; Dányi, Tuf, 2012: 27; Dyachkov, 2017b: 454; Nefediev et al., 2017a: 221; 2017b: 116; 2018: 233.

MATERIAL. East Kazakhstan Region: $10^{7}, 1$ ㅇ (ASU No. 87), Altai Mts, Listvyaga Mt. Range, near Aksharbak village, N49 ${ }^{\circ}$ 31'59.66", E085'31'59.74", 1400 m a.s.1., 3-5.VII.2018; 3 O $^{77}, 2$ +9 , (ASU No. 88), same Region, Saur Mts, Tas Mt. Range, northern slope, $\mathrm{N} 47^{\circ} 16^{\prime} 00.10^{\prime \prime}$, $\mathrm{E} 085^{\circ} 04^{\prime} 00.07^{\prime \prime}, 2230-2400 \mathrm{~m}$ a.s.1., 21-23.VI.2018, all RY, VR, AN.

DISTRIBUTION. Kazakhstan: East Kazakhstan and Almaty regions [Farzalieva, 2006], Russia: Omsk Region [Nefediev et al., 2017b], Altai Province and the Republic of Altai [Dyachkov, 2017b; Nefediev et al., 2017a, 2018]. 

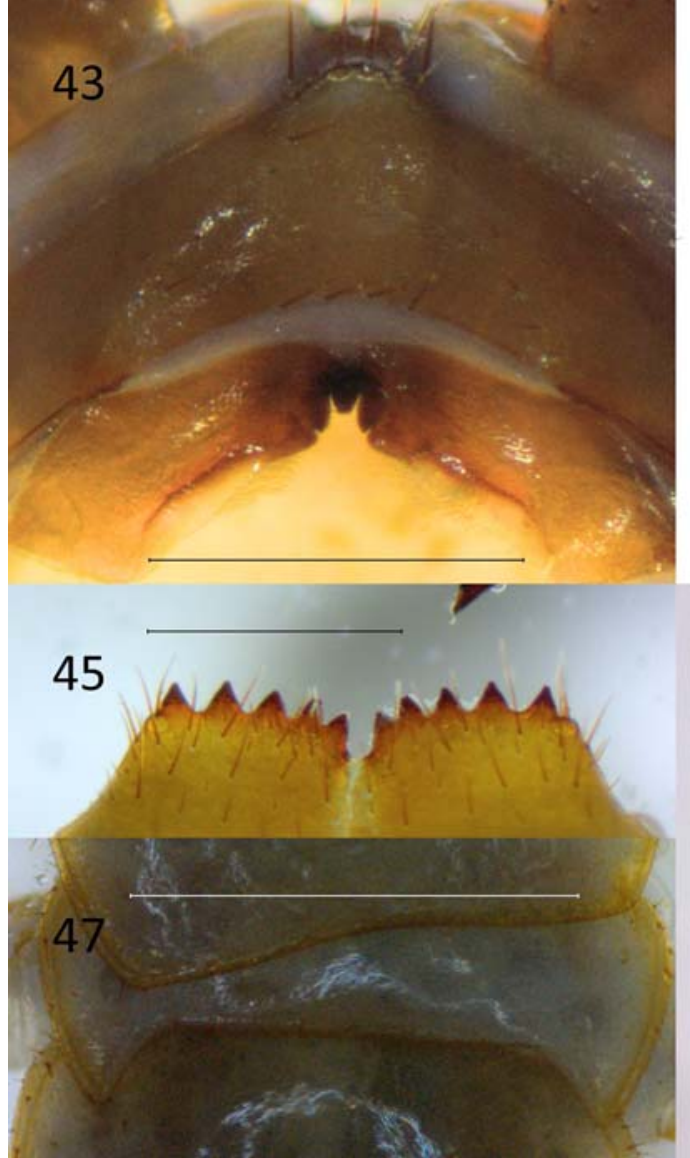
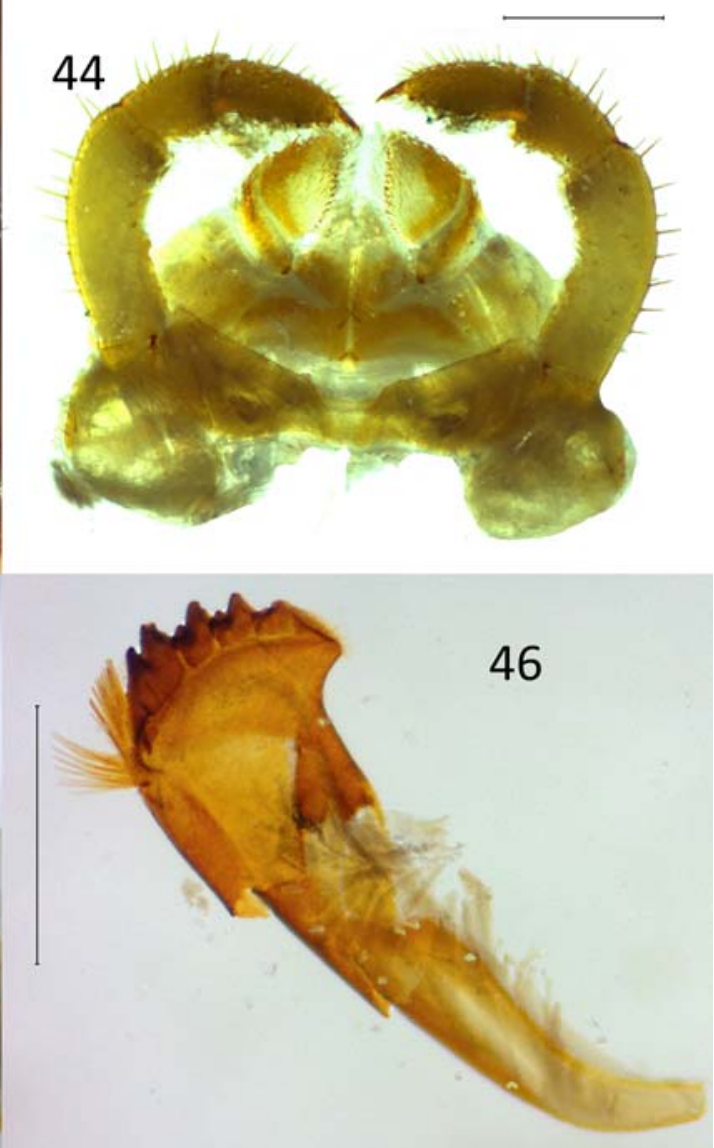

Figs 43-47. Lithobius forficatus (Linnaeus, 1758): 43 — clypeus and labrum, ventral view; 44 - maxillary complex, ventral view; 45 dental margin of forcipular coxosternite, ventral view; 46 - mandible; 47 - tergite 11, dorsal view. Scale: $43-46-0.5 \mathrm{~mm}, 47-1 \mathrm{~mm}$.

Рис. 43-47. Lithobius forficatus (Linnaeus, 1758): 43 - клипеус и лабрум, вентрально; 44 - максиллярный комплекс, вентрально; 45 - зубной край коксостернита ногочелюсти, вентрально; 46 - мандибула; 47 - тергит 11, дорсально. Масштаб: $43-46-0,5$ мм, $47-1$ мм.

REMARKS. Clypeus, labrum, maxillary complex, mandible, dental margin of forcipular coxosternite and 14 leg of male as in Figs 52-56. This species is new to the fauna of the Saur Mts.

\section{Conclusions}

At present, the lithobiomorph fauna of Kazakhstan comprises 34 species from 3 families (Anopsobiidae, Henicopidae, Lithobiidae), all distributed mostly in the mountains in the eastern and southern parts (Table).

Acknowledgments. I am most grateful to S.I. Golovatch (Moscow, Russia) who kindly checked the English of an advanced draft and to G.Sh. Farzalieva (Perm, Russia) for her help in preparing this paper. I wish to also thank all collectors who donated their material for study: A.A. Fomichev, R.V. Yakovlev, A.E. Naydenov, E.A. Nepaeva (all from Barnaul, Russia), G.Sh. Farzalieva, I.I. Temreshev (Almaty, Kazakhstan), V.V. Rudoi (Shemonaikha, Kazakhstan), G.N. Kuftina (St. Petersburg, Russia), A.E. Nakonechniy (Novosibirsk, Russia). The author is most thankful to R.V. Yakovlev (Barnaul, Russia), S.V. Kornev (Orenburg, Russia), D.F. Shovkoon (Samara, Russia) and I.I. Temreshev who organized my field trips to eastern and southern Kaza- khstan. My sincerest thanks go to the administrations and scientific departments of the Sayram-Ugam National Park, Syrdarya-Turkestan State Regional Natural Park (both Shymkent, Kazakhstan), Karatau Nature Reserve (Kentau, Kazakhstan) and Kolsay Lakes National Park (Saty, Kazakhstan) for their assistance in and contribution to my field work. I wish to thank M.M. Silantieva, Dean of the Faculty of Biology of the ASU, as well as M.Yu. Solomonova, staff member of the Department of Botany (all from Barnaul) for their logistic help.

\section{References}

Attems C. 1904. Central- und hoch-asiatische Myriopoden // Zoologische Jahrbücher, Abteilung für Systematik. Bd.20. P.113130.

Attems C. 1907. Myriopoden aus der Krim und dem Kaukasus // Arkiv för zoologi. Bd.3. No.25. S.1-16.

Bonato L., Edgecombe G.D., Lewis J.G., Minelli A., Pereira L.A., Shelley R.M., Zapparoli M. 2010. A common terminology for the external anatomy of centipedes (Chilopoda) // ZooKeys. Vol.69. P.17-51.

Bonato L., Chagas Junior A., Edgecombe G.D., Lewis J.G.E., Minelli A., Pereira L.A., Shelley R.M., Stoev P., Zapparoli M. 2016. ChiloBase 2.0 - A World Catalogue of Centipedes (Chilopoda). Available at http://chilobase.biologia.unipd.it. 

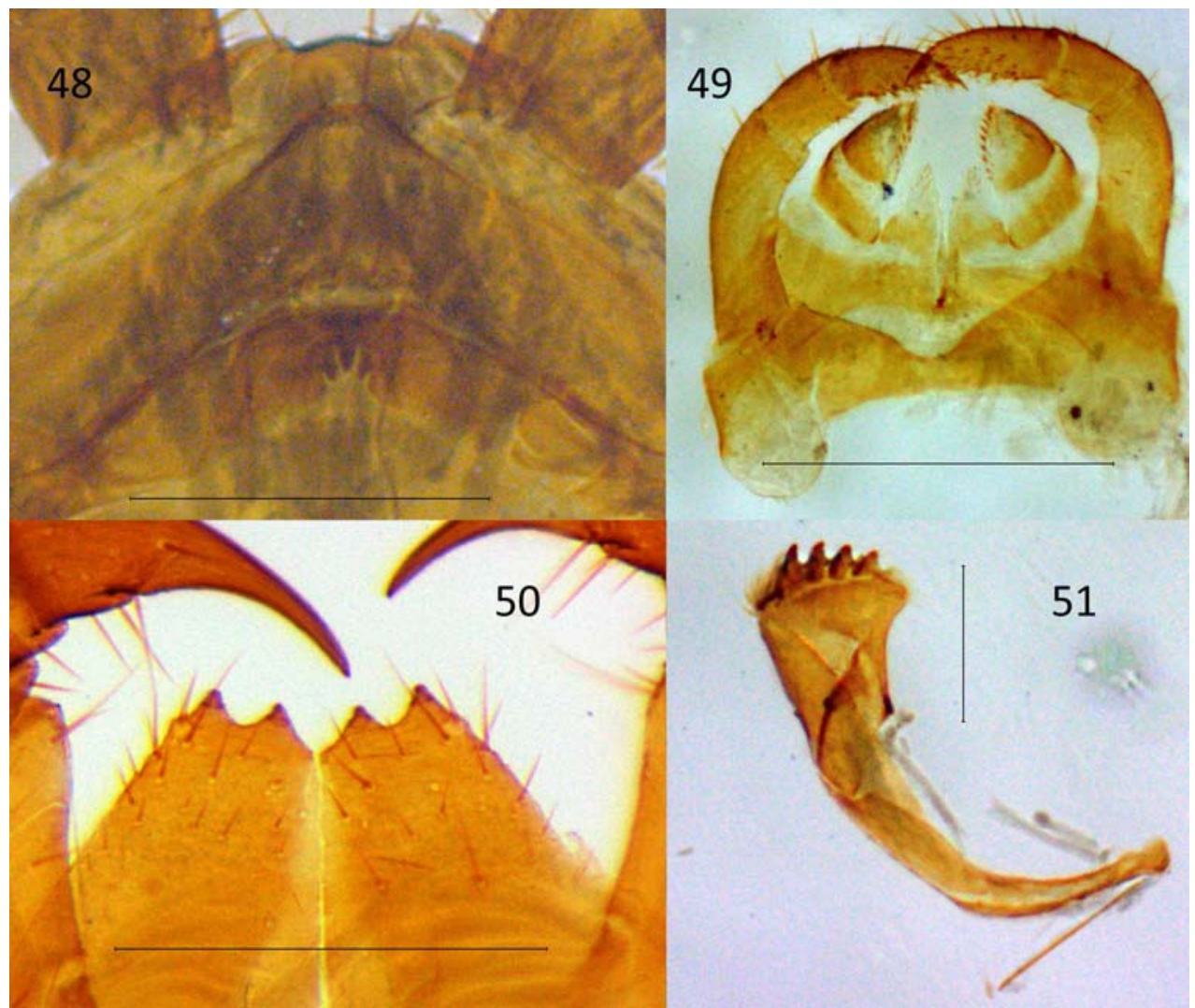

Figs 48-51. Lithobius franciscorum Dányi et Tuf, 2012: 48 - clypeus and labrum, ventral view; 49 - maxillary complex, ventral view; 50 - dental margin of forcipular coxosternite, ventral view; 51 - mandible. Scale: $48,49-0.5 \mathrm{~mm}, 50-0.25 \mathrm{~mm}, 51-0.12 \mathrm{~mm}$.

Pис. 48-51. Lithobius franciscorum Dányi et Tuf, 2012: 48 - клипеус и лабрум, вентрально; 49 - максиллярный комплекс, вентрально; 50 - зубной край коксостернита ногочелюсти, вентрально; 51 - мандибула. Масштаб: 48, 49 - 0,5 мм, $50-0,25$ мм, $51-0,12$ мм.

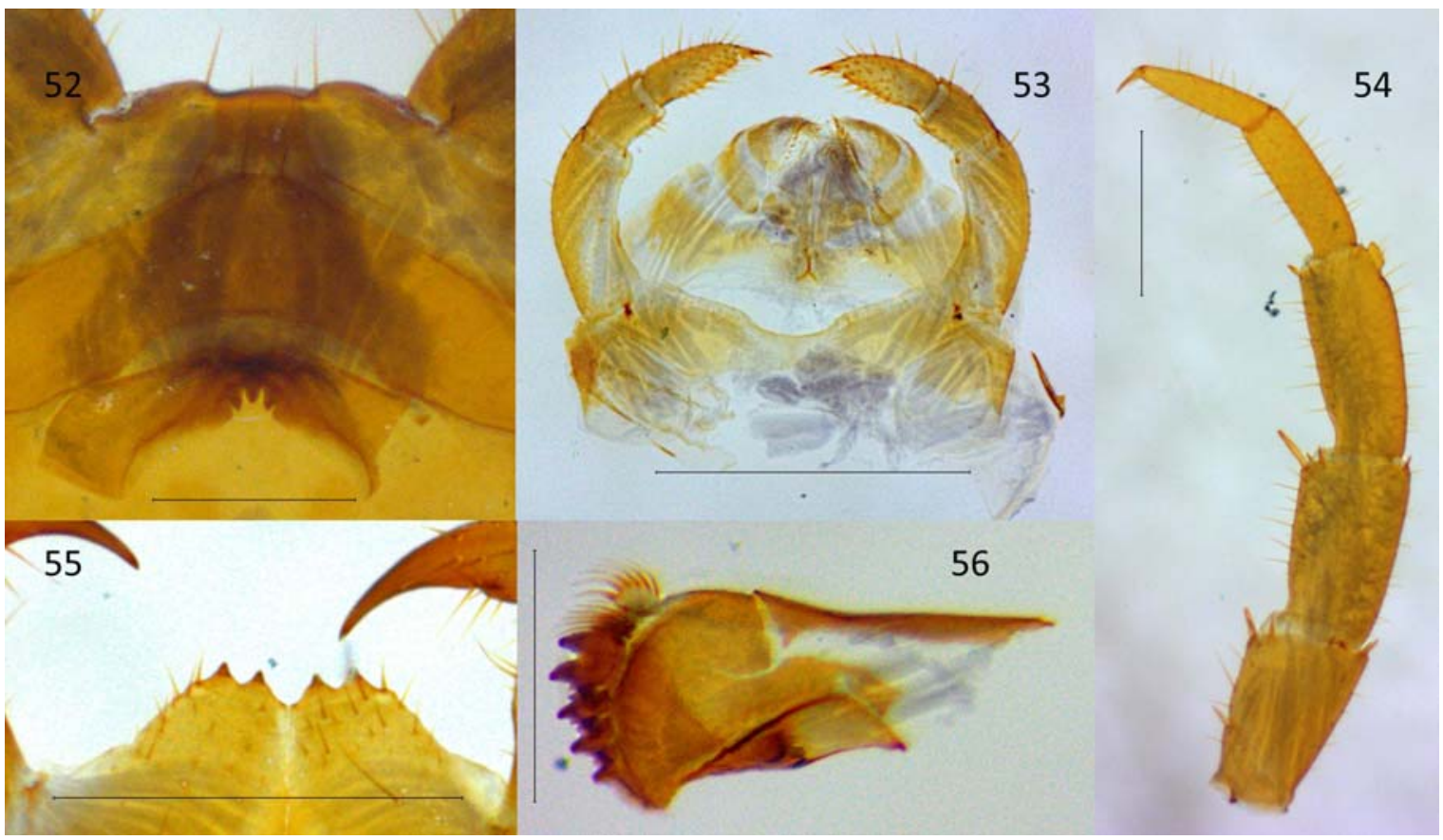

Figs 52-56. Lithobius insolens Dányi et Tuf, 2012: 52 — clypeus and labrum, ventral view; 53 - maxillary complex, ventral view; 54 — leg 14 of male, lateral view; 55 - dental margin of forcipular coxosternite, ventral view; 56 - mandible. Scale: $52-55-0.25 \mathrm{~mm} ; 56-0.12 \mathrm{~mm}$.

Pис. 52-56. Lithobius insolens Dányi et Tuf, 2012: 52 - клипеус и лабрум, вентрально; 53 - максиллярный комплекс, вентрально; 54 - нога 14 самца, латерально; 55 - зубной край коксостернита ногочелюсти, вентрально; 56 - мандибула. Масштаб: $52-55-0,25$ мм; $56-0,12$ мм. 


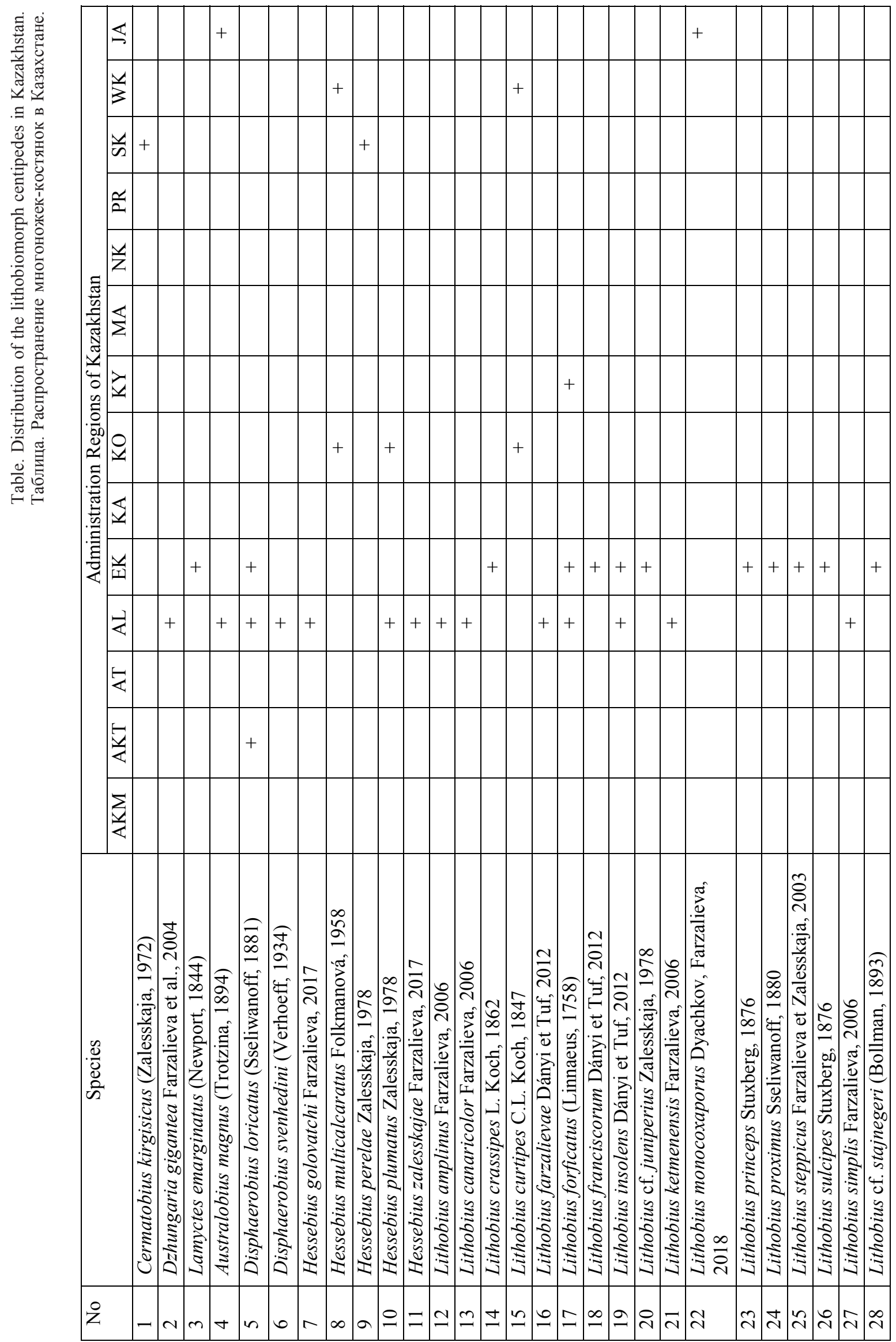




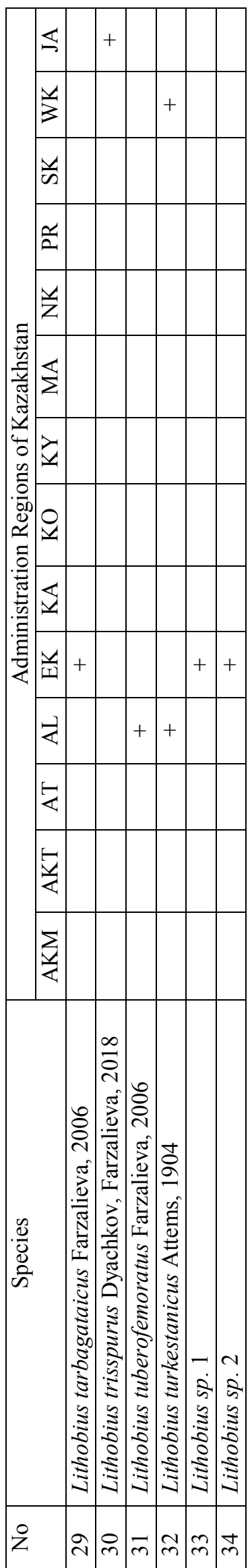

Bragina T.M. 2012. [An inventory of the invertebrate fauna of the Naurzum Nature Reserve] // Material of the II International Scientific Conference "Biodiversity of Asian steppes", Kostanay. P.140-145 [in Russian].

Bragina T.M. 2016. Soil macrofauna (invertebrates) of Kazakhstanian Stipa lessingiana dry steppe // Hacquetia. Vol.15. No.2. P.105-112.

Eason E.H. 1989. Lithobiidae from the Nepal Himalayas with description of ten new species of Lithobius and Australobius // Zoologische Jahrbücher, Abteilung für Systematik. Bd.116. P.355-372.

Eason E.H. 1997. On some Lithobiomorpha from the mountains of Kirghizia and Kazakhstan (Chilopoda) // Arthropoda Selecta. Vol.6. P.117-121.

Dányi L., Tuf I.H. 2012. Lithobius (Monotarsobius) franciscorum sp. nov., a new lithobiid species from the Altai, with a key to the Central Asian species of subgenus (Chilopoda: Lithobiomorpha) // Zootaxa. Vol.3182. P.16-28.

Dyachkov Yu.V. 2017a. [New data on Australobius magnus (Trotzina, 1894) (Chilopoda: Lithobiomorpha: Lithobiidae) from southern Kazakhstan] // Ukrainian Journal of Ecology. Vol.7. No.4. P.440-443 [in Russian with English summary].

Dyachkov Yu.V. 2017b. [The first data on the centipede (Chilopoda: Geophilomorpha; Lithobiomorpha) fauna of the Katunskiy Biosphere State Nature Reserve, Altai Mts] // Ukrainian Journal of Ecology. Vol.7. No.4. P.453-456 [in Russian with English summary].

Dyachkov Yu.V., Farzalieva G.Sh. 2018. Two new species of lithobiid centipedes (Chilopoda: Lithobiomorpha: Lithobiidae) from southern Kazakhstan // Arthropoda Selecta. Vol.27. No.3. P. $210-218$.

Dyachkov Yu.V., Farzalieva G.Sh., Fomichev A.A. 2016. New data on the centipede (Chilopoda) fauna of East Kazakhstan region // Biological Bulletin of Bogdan Chmelnitskiy Melitopol State University. Vol.6. No.3. P.438-442.

Farzaliva G.Sh. 2006. New species of the lithobiid genus Lithobius (Monotarsobius) (Chilopoda: Lithobiomorpha: Lithobiidae) from eastern Kazakhstan // Arthropoda Selecta. Vol.15. No.2. P.99-117.

Farzalieva G.Sh. 2008. [The fauna and chorology of Myriapoda from the Urals and Cisuralia]. PhD Thesis. Perm State University. 189 p. [In Russian]

Farzalieva G.Sh. 2017. [New species of lithobiomorph centipedes of the genus Hessebius Verhoeff, 1941 (Lithobiomorpha, Lithobiidae) from Eastern Kazakhstan] // Zoologicheskii zhurnal. Vol.96. P.30-36 [in Russian, with English summary].

Farzalieva G.Sh., Esyunin S.L. 2008. [A review of the centipede (Lithobiomorpha, Henicopidae, Lithobiidae) fauna of the Urals and Cis-Ural Area] // Zoologicheskii zhurnal. Vol.87. No.8. P.923-947 [in Russian with English summary].

Farzalieva G.Sh., Nefediev P.S., Tuf I.H. 2017. Revision of Disphaerobius Attems, 1926 (Chilopoda: Lithobiomorpha: Lithobiidae: Pterygoterginae), a centipede genus with remarkable sexual dimorphism // Zootaxa. Vol.4258. No.2. P.121-137.

Farzalieva G.Sh., Zalesskaja N.T. 2003. On two remarkable species of lithobiid centipedes (Chilopoda: Lithobiomorpha: Lithobiidae) from the steppe of the southern Urals, Russia // Arthropoda Selecta. Vol.11. No.4. P.265-269.

Farzalieva G.Sh., Zalesskaja N.T., Edgecombe G.D. 2004. A new genus and species of lithobiomorph centipede (Chilopoda: Lithobiomorpha: Anopsobiidae) from eastern Kazakhstan // Arthropoda Selecta. Vol.13. No.4. P.219-224.

Folkmanová B. 1958. [New forms of centipedes (Chilopoda) from the USSR] // Zoologicheskii zhurnal. Vol.37. P.183-192 [in Russian].

Koch M., Edgecombe G.D. 2008. The peristomatic structures of Lithobiomorpha (Myriapoda, Chilopoda): Comparative morphology and phylogenetic significance // Journal of Morphology. Vol.269. P.153-174.

Kunah O.N. 2013. [Chilopoda of the steppe Dnieper area] // Biological Bulletin of Bogdan Chmelnitskiy Melitopol State University. Vol.1. No.7. P.65-81 [in Russian with Ukrainian summary]. 
Lignau N.G. 1929. Zur Kenntnis der zentralasiatischen Myriopoden // Zoologischer Anzeiger. Bd.85. S.159-175.

Ma H., Pei S., Hou X., Zhu T., Wu D., Gai Y. 2014. An annotated checklist of Lithobiomorpha of China // Zootaxa. Vol.3847. No.3. P.333-358.

Nefediev P.S., Farzalieva G.Sh., Tuf I.H. 2017a. A preliminary review of the centipede fauna of the Altai State Nature Biosphere Reserve, southwestern Siberia, Russia (Chilopoda: Lithobiomorpha, Geophilomorpha) // Arthropoda Selecta. Vol.26. No.3. P.217-224.

Nefediev P.S., Farzalieva G.Sh., Tuf I.H., Nedoev Kh. Kh., Niyazov S.T. 2018. Millipede and centipede assemblages on the northern and southern slopes of the lowland Altais, southwestern Siberia, Russia (Diplopoda, Chilopoda) // ZooKeys. Vol.741. P.219-254.

Nefediev P.S., Knyazev S.Yu., Farzalieva G.Sh., Tuf I.H. 2017b. A contribution to the myriapod fauna of the Omsk Area, Siberia, Russia (Myriapoda: Diplopoda, Chilopoda) // Arthropoda Selecta. Vol.26. No.2. P.113-118

Nefediev P.S., Tuf I.H., Farzalieva G.Sh. 2016. Centipedes from urban areas in southwestern Siberia, Russia (Chilopoda). Part 1. Lithobiomorpha // Arthropoda Selecta. Vol.25. No.3. P.257266.

Prado C.C., Triana H.D., Castillo C.C., Tulande-M E. 2018. First records of Lithobius (Lithobius) forficatus (Linnaeus, 1758) and Lithobius (Lithobius) obscurus Meinert, 1872 (Lithobiomorpha, Lithobiidae) introduced to Colombia // Check List. Vol.14. No.3. P.559-568. https://doi.org/10.15560/14.3.559

Shear W.A. 2018. The centipede family Anopsobiidae new to North America, with the description of a new genus and species and notes on the Henicopidae of North America and the Anopsobiidae of the Northern Hemisphere (Chilopoda, Lithobiomorpha) // Zootaxa. Vol.4422. No.2. P.259-283.

Sseliwanoff A.V. 1881. Neue Lithobiiden aus Sibirien und Centralasien // Zoologischer Anzeiger. Bd.4. H.73. P.15-17.

Vsevolodova-Perel T.S. 2009. [Composition of soil populations of clayey semidesert. Ecological and faunistic characterization of soil-dwelling invertebrates] // A.A. Tishkov (ed.). Animals of argillaceous semi desert of trans-Volga Region (synopsis of faunas and ecological characters). Moscow: KMK Scientific Press Ltd. P.137-138 [in Russian].

Trotzina A. 1894. Vier neue Lithobius-Arten aus Centralasien // Horae Societatis Entomologicae Rossicae. Vol.28. P.247-253.

Tuf I.H. 2007. [Diversity of selected taxa of invertebrates in the Altai (East Kazakhstan)] // Modern approaches to biodiversity protection in the context of steady development achievement of the Republic of Kazakhstan. Materials of International Kazakh-Czech Scientific Conference, Ust-Kamenogorsk, 2007. P.56-64 [in Czech, summary in English].

Tuf I.H., Dányi L., Kuda F., Chilachula J. 2010. [Centipedes of Kazakhstan - new records from Altai] // High Mountain Soils Biodiversity. 18-20 October 2010. Ilia State University, Institute of Zoology, Tbilisi. P.11-12.

Tuf I.H., Ivinskis P., Rimðaitë J. 2015. A check-list of the centipedes (Chilopoda) of Lithuania // Zootaxa. Vol.4052. No.3. P.394-400.

Zalesskaja N. 1972. [A new species of the genus Esastigmatobius (Lithobiomorpha, Henicopidae)] // Zoologicheskii zhurnal. Vol.51. P.608-611 [in Russian].

Zalesskaja N.T. 1978. [Identification book of the lithobiomorth centipedes of the USSR]. Moscow: Nauka Publ. 212 p. [In Russian]

Zapporoli M., Edgecombe G.D. 2011. [Order Lithobiomorpha] // A. Minelli (Ed.). Treatise on Zoology-Anatomy, Taxonomy, Biology. The Myriapoda the Myriapoda. Vol. 1. Leiden-Boston: Brill. P.371-389.

Zuev R.V. 2016. Centipedes (Chilopoda) from the Stavropol Territory, northern Caucasus, Russia // Arthropoda Selecta. Vol.25. No.1. P.23-38.

Zuev R.V., Evsyukov A.P. 2016. Centipedes (Chilopoda) from the Rostov-on-Don Region, southern Russia // Russian Entomological Journal. Vol.25. No.4. P.417-426.

Responsible editor S.I. Golovatch 